Rev. Saude puibl., s. Paulo

7:385-41, 1973.

\title{
CICATRIZAÇÃO DE LESÕES CUTÂNEAS DE LEISHMANIOSE AMERICANA COM DOSE UNICA DE PAMOATO DE CICLOGUANIL
}

\author{
Hermelino Herbster GUSMÃO * \\ José Antonio Gomes de SOUZA *
}

RSPU-B/186

Gusmão, H. H. \& SouzA, J. A. G. de - Cicatrização de lesões cutâneas de leishmaniose americana com dose única de pamoato de cicloguanil. Rev. Saúde públ., S. Paulo, 7:335-41, 1973.

Resumo: Relata-se a completa cicatrização de lesões de leishmaniose tegumentar americana, com Pamoato de Cicloguanil (Comolar Parke-Davis) em 33 pacientes, dos quais 28 receberam dose única e 5 dose dupla desse quimioterápico. $O$ diagnóstico foi comprovado pela presença de leishmanias ao exame direto de laboratório. A idade das lesões variou de 8 dias até 12 meses, com $82 \%$ dos casos entre 8 e 30 dias. O tempo médio de cicatrização foi de 104 dias variando entre 60 e 170 dias, para os doentes que receberam apenas uma dose do medicamento. Os doentes que receberam duas doses tiveram a completa cicatrização com tempo médio de 163 dias, após a primeira dose e 85 dias após a segunda. Após a cicatrização, os doentes foram controlados por um periodo médio de 8,4 meses variando entre 3 e 19 meses. A observação mostrou que a cura das lesões é de forma mais retardada, podendo em muitos casos haver uma recrudescência das lesôes e, em alguns casos, uma segunda dose acelera a cura.

UNITERMos: Leishmaniose (quimioterapia) *; Cicloguanil * Terapêuticc* *.

I N T R O D U C A O

No periodo de novembro de 1969 a abril de 1971, a Divisão de Saúde da ICOMI (Indústria e Comércio de Minérios) no Território Federal do Amapá tratou e controlou, constatando a completa cicatrização das lesões, 28 casos de leishmaniose tegumentar americana com dose única de pamoato de cicloguanil, conhecido anti-malárico de longa duração, comercializado sob o nome de "Camolar", pelo Laboratório Park Davis.
Outros 5 casos foram igualmente curados, porém, com duas doses do mesmo quimioterápico.

No mesmo período foram tratados e curados outros quatro casos que não constam da análise e das tabelas que se seguem, porque o exame direto de laboratório foi negativo para pesquisa de Leishmania, apesar das lesões serem típicas e da prova terapêutica.

- Da Indústria e Comércio de Minérios S. A. - ICOMI - Av. Graça Aranha, 26 - Rio de Janeiro, GB - Brasil. 
GUSMão, H. H. \& SoUzA, J. A. G. de - Cicatrização de lesôes cutâneas de leishmaniose americana com dose única de pamoato de cicloguanil. Rev. Saúde públ., S. Paulo, 7:335-41, 1973.

Finalmente, outros 19 casos de leishmaniose cutânea receberam a dose única de pamoato de cicloguanil, mas não voltaram à unidade de saúde da ICOMI para o controle dos resultados. Trata-se de caboclos que moram e trabalham na mata ou ao longo dos rios do interior do Amapá, em locais de difícil controle. Pela falta de comparecimento aos exames após o tratamento, esses 19 casos foram também excluidos da análise que se segue. Entretanto, ainda que se admitisse que todos os 19 casos não controlados não se houvessem curado, vale relatar que os 33 pacientes acompanhados tiveram cura completa.

Peña Chavarria et al. 3,4 $(1965,1968)$ relataram bons resultados no tratamento com dose única de pamoato de cicloguanil de casos de leishmaniose cutânea americana, em Costa Rica. Em 1966, BeLtraN et al. ${ }^{1}$ relataram cura rápida (2 a 4 semanas) de lesões crônicas de leishmaniose cutânea mexicana, usando igualmente uma dose intramuscular de pamoato de cicloguanil, em suspensão oleosa que acarreta lenta absorção da droga.

Em 1968, Johnson 2 e Walton et al. 5 relatam resultados igualmente favoráveis com o mesmo tipo de tratamento para casos de leishmaniose cutânea americana no Panamá. Esses últimos 5 assinalam, entretanto, peculiaridades diferentes dos casos de Costa Rica e México, a saber:

a) a cura das lesões provocadas por Leishmania brasiliensis se manifesta de forma mais retardada, após periodo de 4 a 8 ou mais semanas em seguida à injeção;

b) em muitos casos, nas primeiras semanas, antes de se iniciar a regressão e cicatrização, observa-se uma recrudescência das lesões; c) em alguns casos uma segunda injeção após 6 a 8 semanas, acelerou a cura.

Essas características assinaladas por JoHNSON 2 e WALTON ${ }^{5}$ coincidem com a observação dos casos tratados no Amapá, aqui relatados.

\section{IDADE DAS LESOEES ANTES DO TRATAMENTO}

O caso 2 apresentou a lesão mais antiga, já registrada no hospital 12 meses antes do tratamento com uma dose de pamoato de cicloguanil.

Excetuados ainda os casos de números $1,7,13,27$ e 32, cujas lesões eram conhecidas, respectivamente, há $2,4,2,2$ e 3 meses antes do início do tratamento, todos os demais pacientes $(82 \%$ dos casos tratados) relataram haver notado o aparecimento das lesões entre 8 e 30 dias antes do tratamento.

Trata-se, pois, de uma série de casos cujas lesões eram bastante recentes. $O$ tempo de duração das lesões de cada caso, antes do tratamento é apresentado na coluna "Início da Lesões" da Tabela, que sumariza o protocolo dos casos tratados.

\section{TEMPO DE CICATRIZAÇÃO APÓS DOSE} UNICA DE PAMOATO DE CICLOGUANIL

Os critérios de cura clínica e cura parasitológica da leishmaniose tegumentar variam de acordo com as facilidades de laboratório e com as possibilidades de freqüente observação clínica dos pacientes. Para a presente observação, preferimos limitar a avaliação de eficácia terapêutica à verificação clínica da cicatrização e epitelização das lesões leishmanióticas, registrando o tempo decorrido entre a administração da droga e a constatação dessa cicatrização. 
GUSMAO, H. H. \& SOUZA, J. A. G. de - Cicatrização de lesర̋es cutâneas de leishmanlose americana com dose única de pamoato de cicloguanil. Rev. Saúde Públ., S. Paulo, 7:335-41, 1973.

Por outro lado, muitos casos apresentaram exames de laboratório negativos para pesquisa direta de leishmaniose (4 ou mais semanas após a injeção) com úlceras ainda francamente abertas.

Originalmente foi programado um controle mensal dos pacientes tratados. Entretanto, isto não foi possível na prática, portanto o comparecimento foi irregular. Em alguns casos, os prazos mais longos de cicatrização registrados (4 e 5 meses) representam prazos máximos pois, por vezes, os pacientes passaram dois e até três meses sem comparecer ao hospital. Em consequiência, quando o paciente era examinado, já apresentava cicatrização completa há muitos dias. Dos 33 casos tratados e curados, para cálculo de tempo de cicatrização, foram postos de lado sete casos, a saber:

a) o caso 6 não entrou no cálculo do tempo médio de cicatrização, porque só voltou ao exame de controle 11 meses após a dose única do quimioterápico, se bem que com completa cicatrização de 27 úlceras leishmanióticas. Não foi assim possivel estabelecer quando, anteriormente ao exame do $11 .^{\circ}$ mês, ocorreu cicatrização de todas essas lesões;

b) o caso 30 não foi computado, porque a descrição da involução da lesão, no controle de 30 dias após o tratamento, faz menção a uma precoce epitelização da úlcera, sem precisar se havia cicatrização completa da lesão. Posteriormente, esse paciente só foi visto aos seis meses e meio, com a lesão completamente cicatrizada e epitelizada.

c) postos de lado ainda os cinco casos que receberam uma segunda dose da droga e que estão analisados à parte, o prazo de cicatrização para os 26 pacientes restantes, teve os seguintes resultados:
1) oito casos $(30,77 \%)$ tiveram a cicatrização das lesões constatada entre 60 e 89 dias, isto é, no decorrerer do terceiro mês de observação.

2) nove pacientes $(34,61 \%)$ apresentaram cicatrização de suas lesões no período entre 90 e 119 dias, ou seja, no decurso do quarto mês, após a injeção da dose terapêutica única;

3) finalmente, 4 casos $(15,38 \%)$ e 5 casos $(19,23 \%)$ tiveram cicatrização completada entre, respectivamente, 120 e 149 dias e 150 e 170 dias. Isto é, 9 casos $(34,61 \%)$ só completaram a cicatrização decorridos mais de 4 meses após a dose única terapêutica;

4) esses 26 casos apresentaram um tempo acumulado de 90 meses e 10 dias para a cicatrização de todas as suas lesões, o que representa um periodo médio, por paciente, de 3 meses e duas semanas, ou seja, um total de 104 dias, para a cura completa.

TEMPO DE CICATRIZAÇA DAS LESOES LEISHMANIOTICAS APOS A DOSE UNICA DE PAMOATO DE CICLOGUANIL

$\begin{array}{lcc}\text { P e r fod os } & \text { Casos } & \% \\ \text { De } 60 \text { a } 89 \text { dias } & 8 & 30,77 \\ \text { De } 90 \text { a } 119 \text { dias } & 9 & 34,61 \\ \text { De } 120 \text { a } 149 \text { dias } & 4 & 15,38 \\ \text { De } 150 \text { a } 170 \text { dias } & 5 & 19,23 \\ \text { Total } & 26 & \end{array}$

TEMPO DF CICATRIZACAO COM DUAS INJEÇOES DE PAMOATO DE CICLOGUANIL

Em cinco (casos 4, 7, 16, 19 e 21) dos 33 casos controlados, foi administrada uma segunda dose do quimioterápico, sendo que em três casos a segunda injeção foi dada 3 meses após a primeira e, nos dois outros casos, 2 meses após a dose inicial. 


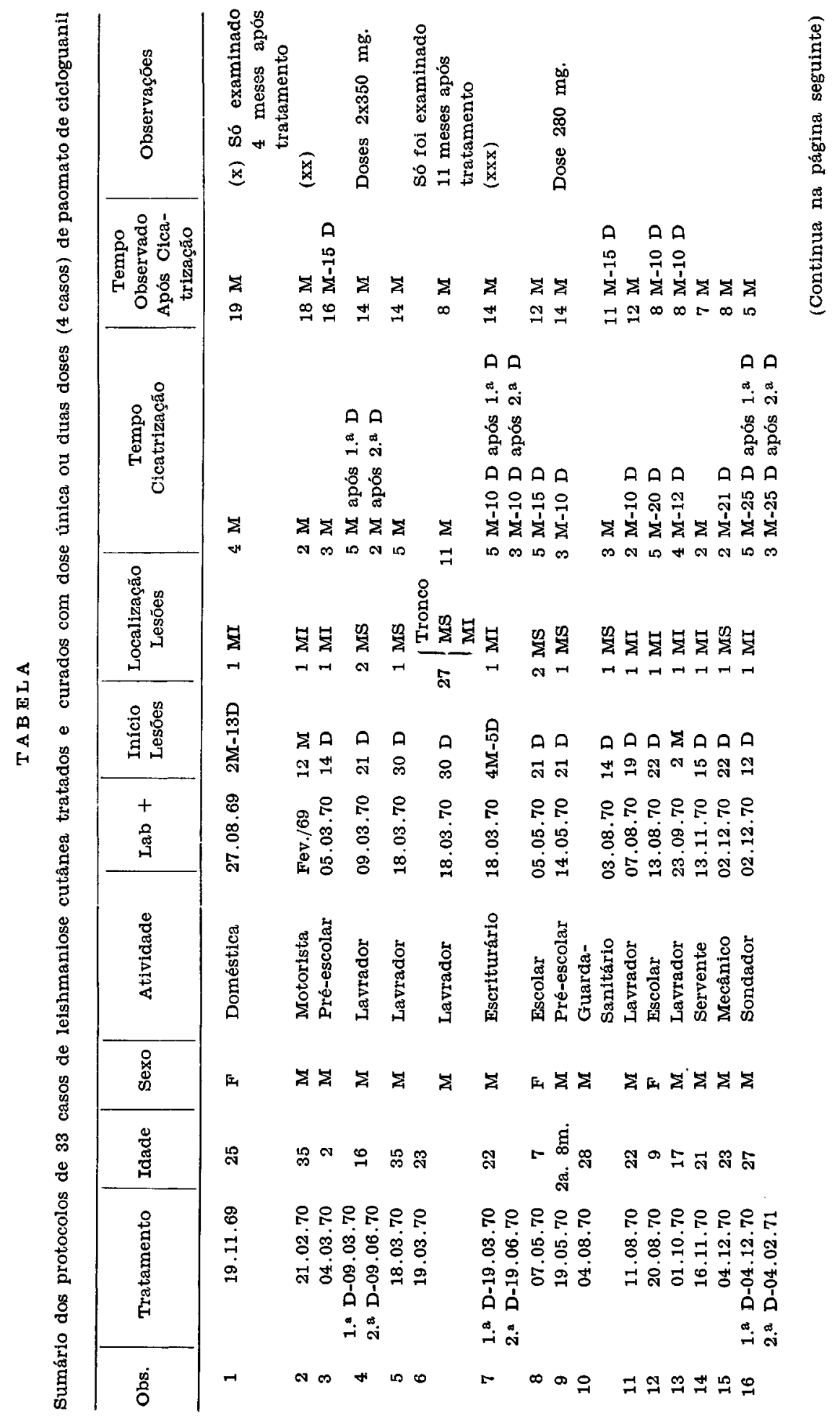




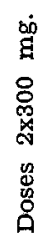

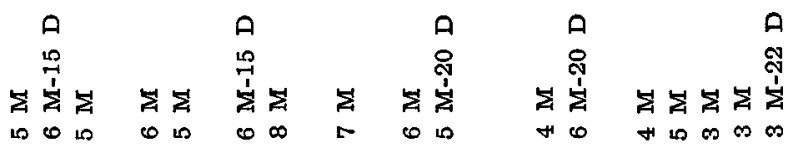

คคคค

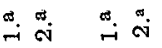

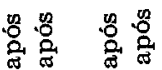

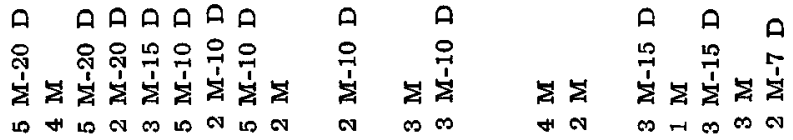

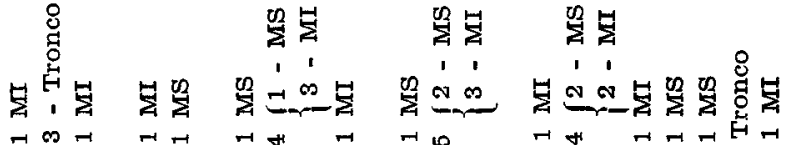

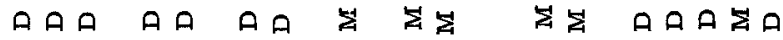

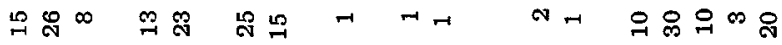

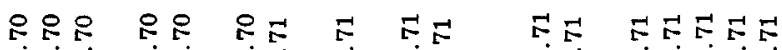
ำ ำ ํํㅇ ㅎํㅇ

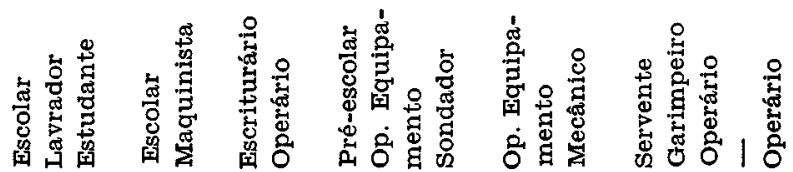

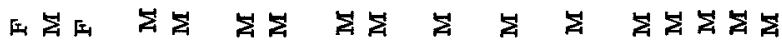
윽 สㄷㄷ

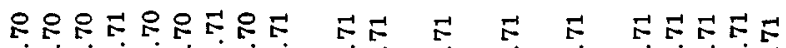

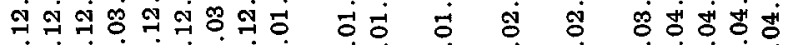
ப்

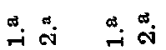

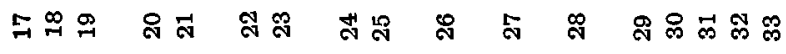


Gusmao, H. H. \& SoUzA, J. A. G. de - Cicatrização de lesóes cutâneas de leishmaniose americana com dose única de pamoato de cicloguanil. Rev. Saúde públ., S. Paulo, 7:335-41, 1973.

A razão dessa tentativa de uma nova dose da medicação, foi a observação de que as lesões continuaram ativas, com aparecimento de novos nódulos que se ulceravam ao longo dos trajetos linfáticos próximos às lesões originais, ao fim de 2 e 3 meses de observação, após a primeira injeção. $\mathrm{Na}$ realidade, vários casos nas 8 ou 10 semanas que se seguiram à primeira injeção, sofreram uma visível reação ativadora da lesão, antes de iniciar o periodo de involução.

Em todos os cinco casos a segunda dose da medicação foi seguida de sensível regressão da atividade das lesões num período de 6 a 10 semanas.

O tempo médio de cicatrização desses casos foi de 163 dias ou 5 meses e 13 dias após a primeira injeção e 85 dias ( 2 meses e 25 dias) após a segunda dose.

\section{CASOS TRATADOS ANTERIORMENTE COM ANTIMONIAL PENTAVALENTE (GLUCANTIME)}

Três (casos 1, 2 e 7) dos 33 casos controlados, receberam tratamento com antimonial pentavalente (glucantime), antes de tomarem a dose única de camolar.

O caso um tomou 50 ampolas de glucantime, com melhora parcial, continuando a lesão aberta e em atividade. Quatro meses após a dose única de pamoato de cicloguanil, a lesão estava cicatrizada e epitelizada. Um exame de controle, 19 meses após a primeira verificação de cura, revelou que a lesão permanecia cicatrizada, sem sinal de recidiva. Entre a última injeção de antimonial e a dose de pamoato de cicloguanil, decorreram 2 meses.

O caso 2 apresentava úlcera leíshmaniótica 12 meses antes da dose única de pamoato de cicloguanil. Durante esse período o paciente, segundo sua afirma- ção, fez várias séries de injeções de glucantime num total de 232 ampolas. Foi feita, também sem resultado prático, uma cauterização da lesão. Dois meses após a dose única de pamoato de cicloguanil, a lesão estava cicatrizada e epitelizada. Um controle, 18 meses após a cura, constatou persistência de completa cicatrização, sem qaulquer recidiva. A dose de pamoato de cicloguanil foi dađa um mês após a última injeção de antimonial pentavalente dos tratamentos anteriores.

O caso 7 conhecia a lesão 4 meses e 5 dias antes do tratamento com pamoato de cicloguanil. Durante esse tempo tomou 60 ampolas de glucantime, em séries de 10 dias. Em virtude da persistência de úlcera ativa, foi feita resseção cirúr. gica, também sem cicatrização. Um mês após a última injeção de glucantime, foi dada uma dose de $350 \mathrm{mg}$. de pamoato de cicloguanil.

Três meses após, permanecendo aberta e ativa a lesão, foi feita uma segunda dose de pamoato de cicloguanil. A completa cicatrização da lesão foi constatada 5 meses e 10 dias após a primeira e 3 meses e 10 dias após a segunda dose de pamoato de cicloguanil.

Um controle, 14 meses após a cura, confirmou cicatrização sem ocorrência de recidiva.

\section{TEMPO DE OBSERVAÇAO APÓS A CICATRIZAÇÁO}

No total dos 33 casos curados, houve um seguimento de 277 meses e 12 dias, ou seja, a média de 8,4 meses por caso, após a cicatrização das lesões, sem ocorrência de recidiva.

Dez casos foram observados em períodos de 11 a 19 meses após a constatação da cicatrização, sem que fosse verificada 
GUSMAO, H. H. \& SOUZA, J. A. G. de - Cicatrização de lesóes cutâneas de leishmaniose americana com dose única de pamoato de cicloguanil. Rev. Saude públ., S. Paulo, 7:335-41, 1973.

qualquer ocorrência de recidiva das lesões.

Doze casos foram observados entre 6 e 8 meses, igualmente sem sinal de recidiva.
Finalmente, um grupo de 11 casos, de tratamento mais recente, teve um seguimento entre 3 e 5 meses após a cicatrização, sem recidiva.

GuSMão, H. H. \& SouzA, J. A. G. de - [Cicatrization of lesions of cutaneous leishmaniasis with a single dose of cicloguanil pamoate.] Rev. Saúde públ., S. Paulo, 7:335-41, 1973.

Summary: The treatment of Cutaneous Leishmaniasis with Cicloguanil Pamoate ("Comolar" Park-Davis), in Amapa Territory, North of the Amazon River, Brazil, is reported. Complete cicatrization of lesions was observed in 33 patients of which 28 received a single dose and 5 were treated with a second injection of the drug. Diagnosis was made by direct microscopic examination and the finding of Leishmaniae. Age of the lesions varied from 8 days to 12 month, $82 \%$ being from 8 to 30 days $s_{+}$The average time necessary for cicatrization was 104 days, varying from 60 to 170 days for patients receiving a single dose. Complete cicatrization among patients that received a second dose occurred in an average time of 163 days after the first treatment and 85 days after the second dose. After cicatrization the patients were kept under observation for an average period of 8.4 months, varying from 3 to 19 months. It was shown that the cure of the lesions requires a rather long period after the first dose, with recrudescence in several cases and requiring in some patients a second dose to accelerate the cure.

UNITERMS: Leishmaniasis, cutaneous *; Chemotherapy *; Cycloguanil pamoate *

\section{REFERENCIAS BIBLIOCRAFTCAS}

1. BELTRAN, D. et al. - Tratamiento de la leishmaniosis cutanea mexicana com Camolar (CI-501) - Pren. méd. mex., 31:365-8, 1968.

2. Johnson, c. - Cycloguanil Pamoate in the treatment of cutaneous lelshmaniasis: initial trials in Panama. Amer. J. trop. Med. Hyg., 17:819-22, 1968.

3. PEifa Chavarria, A. et al. - Preliminary evaluation of Cycloguanil Pamoa- te in dermal leishmaniasis. J.A.M.A., 194:240-2, 1965.

4. PENA ChavarRIA, A. et al. Treatment of American dermal leishmaniasis with Cycloguanil Pamoate. Trans. roy. soc. trop. Med. Hyg., 62:550-5, 1968.

5. WALTON, B. et al. - Treatment of American cutaneous leishmaniasis with Cycloguanil Pamoate. Amer. J. trop. Med. Hyg., 17:814-8, 1968.

Recebido para publicação em 20-8-1973. Aprovado para publicaçăo em 9-10-1793. 